\title{
Sites of Plasticity in the Neural Circuit Mediating Tentacle Withdrawal in the Snail Helix aspersa: Implications for Behavioral Change and Learning Kinetics
}

\author{
Steven A. Prescott ${ }^{1}$ and Ronald Chase \\ Department of Biology, McGill University \\ Montreal, Quebec, H3A 1B1, Canada
}

\begin{abstract}
The tentacle withdrawal reflex of the snail Helix aspersa exhibits a complex combination of habituation and sensitization consistent with the dual-process theory of plasticity. Habituation, sensitization, or a combination of both were elicited by varying stimulation parameters and lesion condition. Analysis of response plasticity shows that the late phase of the response is selectively enhanced by sensitization, whereas all phases are decreased by habituation. Previous data have shown that tentacle withdrawal is mediated conjointly by parallel monosynaptic and polysynaptic pathways. The former mediates the early phase, whereas the latter mediates the late phase of the response. Plastic loci were identified by stimulating and recording at different points within the neural circuit, in combination with selective lesions. Results indicate that depression occurs at an upstream locus, before circuit divergence, and is therefore expressed in all pathways, whereas facilitation requires downstream facilitatory neurons and is selectively expressed in polysynaptic pathways. Differential expression of plasticity between pathways helps explain the behavioral manifestation of depression and facilitation. A simple mathematical model is used to show how serial positioning of depression and facilitation can explain the kinetics of
\end{abstract}

\footnotetext{
${ }^{1}$ Corresponding author. Present address: Department of Pharmacology and Therapeutics, McGill University, Montreal, Quebec, H3G 1Y6, Canada.
}

dual-process learning. These results illustrate how the position of cellular plasticity in the network affects behavioral change and how forms of plasticity can interact to determine the kinetics of the net changes.

\section{Introduction}

An important goal in studies of learning and memory is to relate cellular plasticity to behavioral change. To achieve this, one must consider what is happening at the neural network level. The importance is twofold. First, different network elements may mediate different components of the behavioral response, meaning that plasticity in different network elements will confer learning in different components of the behavior. Second, relating learning kinetics at the cellular level to learning kinetics at the behavioral level may be complicated by interactions between learning processes at the network level. The tentacle withdrawal reflex of the snail Helix aspersa is plastic (Prescott and Chase 1996) and is mediated by a relatively simple neural network (Prescott et al. 1997), which makes it a suitable preparation in which to investigate the relationship between cellular plasticity and behavioral change.

Our first goal was to localize plasticity within the neural network to help relate cellular plasticity to behavioral change. We have shown previously that excitation caused by a brief mechanical stimulus to the tip of the tentacle is transformed into a prolonged neuronal discharge in interneurons of the tentacle ganglion (Prescott et al. 1997). Prolonged activity is transmitted through the polysynaptic pathway and is thought to be a major determinant of muscle response duration based on correlative data and direct motoneuron stimulation

LEARNING \& MEMORY 6:363-380 @ 1999 by Cold Spring Harbor Laboratory Press ISSN1072-0502/99 \$5.00

$$
\begin{array}{lllllllllllllll}
L & E & A & R & N & I & N & G & \underset{363}{\boldsymbol{Z}} & M & E & M & O & R & Y
\end{array}
$$


experiments in Helix (Prescott et al. 1997) and modeling studies in Aplysia (Lieb and Frost 1997). Neural activity in the monosynaptic pathway consists of a phasic burst that is rapidly transmitted to the muscle, thereby mediating the early phase of the muscle response. Similar dichotomous roles are seen in the parallel monosynaptic and polysynaptic pathways that mediate withdrawal reflexes in Aplysia (Cleary and Byrne 1993; White et al. 1993; Frost and Kandel 1995; Lieb and Frost 1997). Based on the different functions of the neural pathways, the current study investigated whether differential expression of depression and facilitation in the monosynaptic and polysynaptic pathways can explain why habituation largely determines response amplitude, whereas sensitization largely determines response duration, as previously described in Helix (Prescott and Chase 1996).

We also sought to localize sites of plasticity to investigate the interactions between learning processes. In certain systems, habituation and sensitization can develop concurrently and compete to determine behavioral change and thereby produce a pattern of learning (referred to here as dual-process learning) consistent with the dual-process theory of plasticity proposed by Groves and Thompson (1970). Habituation and sensitization are often considered opposing processes and, therefore, mutually exclusive. But these forms of learning do occur together and work toward a common goal. Sensitization increases the salience of strong and/or novel stimuli (high informational value), whereas habituation decreases the salience of repeated stimuli (low informational value) that, as Brown (1998) points out, serves to further enhance the salience of less frequent, high information stimuli. The combination of sensitization and habituation essentially works toward making behavior effective but not wasteful.

Dual-process learning occurs within diverse systems (for review, see Prescott 1998) including the tentacle withdrawal reflex of Helix (Christoffersen et al. 1981; Zakharov and Balaban 1987; Balaban 1993; Prescott and Chase 1996). The relative positioning of depression and facilitation within the neural network is thought to underlie the learning kinetics that characterize dual-process learning. Thus, the second goal of this paper was to explain the kinetics of dual-process learning by investigating this phenomenon in a relatively simple system.

To achieve the two goals described above, this study made use of the recently improved charac- terization of the neural circuit mediating tentacle withdrawal in the snail Helix aspersa (Fig. 1) (Prescott et al. 1997). Localization of plastic loci was achieved by stimulating and recording at different points in the circuit, thereby including or excluding plastic loci in the stimulus-response pathway. Careful analysis of the responses shows the dissociation of plasticity in the different phases of the response; we relate these changes to differential plasticity in the constituent pathways of the neural circuit. Because habituation and sensitization are differentially influenced by stimulus intensity and repetition, we were able to selectively elicit one or the other form of plasticity by changing stimulation parameters. Habituation is ideally induced by lowintensity, high-frequency (i.e., repetitive) stimulation, whereas sensitization is ideally induced by high-intensity, low-frequency stimulation (Thompson and Spencer 1966). Moreover, in the tentacle withdrawal reflex of Helix, habituation and sensitization are distinguished by their requirements for induction: The central nervous system (CNS) is necessary for the induction of sensitization but is

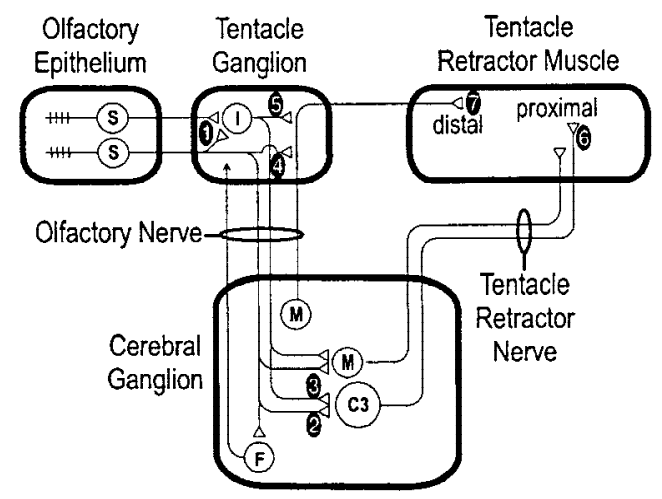

Figure 1: The neural circuit mediating tentacle withdrawal. Mechanical stimulation is applied to the olfactory epithelium. Sensory neurons (S) transmit the neural information through parallel peripheral and central stimulus-response pathways, the latter passing through the cerebral ganglion (CNS). Previous evidence suggests that the short latency response is mediated by monosynaptic pathways, whereas the later, more prolonged response is mediated by polysynaptic pathways. Prolongation of the neural signal probably occurs during transmission through locus 1. Apart from the giant neuron C3, each circle represents a group of cells. Facilitatory neurons (F) have not been identified but are believed to project from the cerebral ganglion into the tentacle ganglion. The numbers indicate potential loci of synaptic plasticity as referred to in the text and in Table 1. (I) Interneurons; $(\mathrm{M})$ motoneurons additional to $\mathrm{C} 3$. Modified from Prescott et al. (1997).

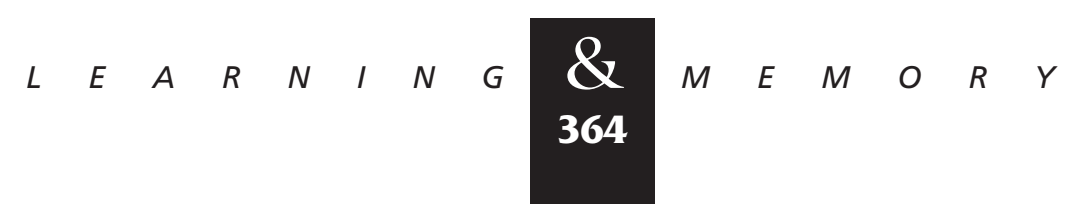


not needed for the induction of habituation, though the peripheral nervous system is sufficient to express both forms of learning (Prescott and Chase 1996). Therefore, we were able to elicit habituation, sensitization, and combinations thereof to obtain the data necessary to test a recently published model of dual-process learning kinetics (Prescott 1998). Some of these data have been published previously in abstract form (S.A. Prescott and R. Chase, unpubl.).

\section{Materials and Methods}

Methodology for the current experiments has been published previously (Prescott and Chase 1996; Prescott et al. 1997); it is summarized here, along with variations and additional information relevant to the current study. Mature specimens of the terrestrial snail Helix aspersa were used for all experiments. Each snail was anesthetized by injection of $\sim 3 \mathrm{ml}$ of isotonic $\mathrm{MgCl}_{2}$. All central nervous ganglia plus the superior tentacles (rhinophores) were removed and dissected in a Sylgard-coated dish filled with a 1:1 mixture of $\mathrm{MgCl}_{2}$ and normal snail saline (Prescott and Chase 1996). For CNS lesions, the olfactory nerve and the tentacle retractor nerve were cut leaving only the tentacle for experimentation. After dissection, the solution was replaced with normal saline, and experimentation was delayed $\geq 30 \mathrm{~min}$.

Mechanical stimulation was effected by a 1-sec pulse of saline applied to the immobilized olfactory epithelium (which under natural conditions is located at the tip of the superior tentacle when the tentacle is extended). The strength of stimulation was adjusted by changing the pump flow rate. Stimulus strength is referred to as weak $(0.23 \mathrm{ml} /$ sec) or strong $(0.41 \mathrm{ml} / \mathrm{sec})$; stimulation stronger than $0.41 \mathrm{ml} / \mathrm{sec}$ was not used in the present study because inhibition can develop after very strong stimulation (Prescott 1997). The tentacle retractor muscle was attached at its proximal end to a force transducer, and its contractile force was measured isometrically. Neural activity was recorded in the olfactory nerve by use of a suction electrode. All olfactory nerve recordings were performed with a CNS lesion because it was necessary to sever the nerve and take up the distal stump in the electrode (as opposed to taking up the nerve en passant) to get an extracellular recording of sufficient quality to clearly identify action potentials. Neural activity was also recorded intracellularly from the identified motoneuron C3. All data were digitized and stored on computer (Digidata 1200 A/D converter and Axotape 2.0.2 software, both from Axon Instruments) for later analysis.

\section{TRAINING SCHEDULE}

The training schedule is adapted from that used previously (Prescott and Chase 1996). The baseline, or naive, response was determined by the first test stimulus (trial 0 ). Blocks of five training stimuli were then applied at 2-min interstimulus intervals; after each block, a single test stimulus was applied. This was repeated five times for a total of 31 stimuli. The responses to the six test stimuli (trials 0-5) constitute the principal data in our analysis. All test stimuli (with one exception described in Results) were "weak" regardless of the stimulus intensity used for training. This allows for a direct comparison of responses to test stimuli such that differences between training conditions are clearly attributable to training, and not to testing. Furthermore, the muscle response to weak stimulation is not saturated at any phase, meaning that sensitization, if present, would not be prevented from causing an observable increase in that phase.

\section{RESPONSE ANALYSIS}

The first $30 \mathrm{sec}$ of each response were recorded and subsequently analyzed to create a response profile. For muscle responses, tension was measured at 0.5 -sec intervals and plotted against time after stimulus onset. For olfactory nerve and C3 responses, action potentials were counted in 0.5 -sec bins and plotted against time after stimulus onset (using time at midpoint of the bin) to give a spike-frequency histogram. This was repeated for trials 0-5. Each measurement in each trial was normalized to the peak response in trial 0 of the same preparation (maximal tension or bin measured over $30 \mathrm{sec}$ ) so that each measurement is expressed as a percentage of that peak. Responses from all preparations used in a given training condition were averaged. Three-dimensional response profiles are used to express the response amplitude at different phases of the response and to show how this changes with repeated stimulation, that is, from trials $0-5$.

Changes in the different phases of the response were quantified in learning curves. For muscle responses, tension was analyzed at three

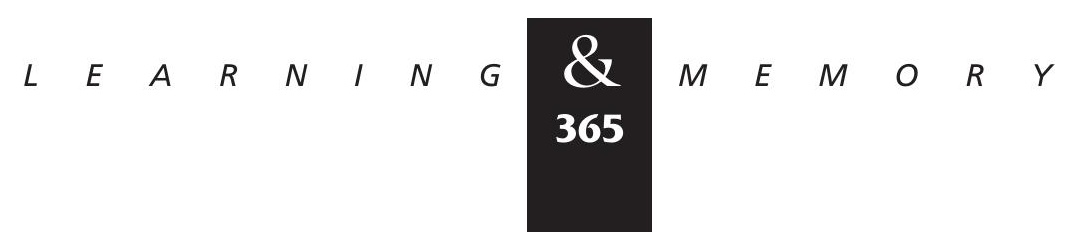


different phases of the response: (1) Rising phase corresponds to tension at $2 \mathrm{sec}$ and reflects the rate of muscle contraction, (2) peak phase corresponds to tension at $7 \mathrm{sec}$ and reflects the peak amplitude of muscle contraction, and (3) late phase corresponds to average tension between 15 sec and $30 \mathrm{sec}$. The average response over $15 \mathrm{sec}$ was analyzed because tension tends to fluctuate during the late phase. Response amplitude during the late phase is taken as an index of response duration because it shows the maintenance of muscle tension and it is not confounded by changes in other phases as can happen with other measures of response duration, for example, changes in peak phase will affect duration at half peak tension. For nerve and $\mathrm{C} 3$ responses, late phase was calculated as above, peak phase corresponds to the time indicated in the appropriate figure legends, and rising phase was not calculated. For each preparation, the response at a particular phase in a given trial is expressed as percent of the response at the same phase in trial 0 . Responses from preparations within the same training condition were averaged. For nerve and C3 responses, $n=3$ preparations; this number is low because of the difficulty in maintaining stable recordings over the duration of training ( $>1 \mathrm{hr}$ ). For muscle responses, $n$ varies for different conditions and is reported in the figure legends. The variation is attributable to the fact that muscle recordings were done simultaneously with nerve or $\mathrm{C} 3$ recordings that were sometimes lost during training; the muscle record was not discarded when the corresponding nerve or $\mathrm{C} 3$ record was incomplete.

Data are reported as mean \pm s.E. For the purposes of statistical testing, data were log transformed after normalization. As a response decreases, further reduction is limited and variance across preparations decreases, whereas the opposite is true as a response increases; a log transformation redistributes the data more evenly and thereby makes variances more similar. Two-tailed unpaired $t$-tests were applied on rising phase and late phase data of each training condition; no tests were applied to peak phase data because changes were generally intermediate compared with the other two phases. Starting with trial 1, testing was repeated until an increasing or decreasing trend reached significance compared with either the baseline response (trial 0 ) in the same training condition or the matching trial in the control condition (weak, infrequent stimulation; Fig. 2B), or until trial 5. A Bonferroni correction was applied based on the number of times $t$-tests were repeated for data of a given phase for a given training condition. Two-way analyses of variance (ANOVAs) were also used to analyze the effects of stimulus intensity and frequency on response plasticity. Statistical analysis and curve fitting were performed on either SigmaStat version 1 or SigmaPlot version 4 (SPSS, Inc.).

\section{Results}

CONTROL CONDITION AND SELECTIVE INDUCTION OF SENSITIZATION

A sample naive muscle response to weak stimulation is shown in Figure 2A. Using stimulation parameters predicted to not cause any plasticity (weak test stimuli at 12-min intervals without any intervening training stimuli), five preparations were tested to verify this prediction and to ensure that the in vitro preparation was stable over the duration of training. Minimal change in the muscle response was observed between trials 0 and 5 (Fig. $2 \mathrm{~B})$. There was some decrease in the rising phase, but the change was not significant compared with baseline nor did the kinetics suggest that habituation was responsible for the change. The increase in the late phase was also not significant, but it may indicate mild sensitization. These data (referred to as "control" later in the text) serve as the standard with which to compare data from other training conditions to demonstrate plasticity in the muscle response.

The purpose of the next experiment was to selectively induce sensitization without habituation by applying strong but infrequent stimuli (at 12-min intervals). This was the only case in which strong stimulation was used for testing; the higher baseline response may cause an underestimation of the percentage increase in the response caused by learning. The muscle response exhibited the effects of sensitization, but not in all phases (Fig. 2C). The late phase response showed the greatest increase, rising to $288 \pm 75 \%$ by trial 1 (which was a significant increase compared with control; $P<0.05$ ) and remaining at this level. The peak phase response was also slightly increased. The rising phase response decreased, but the change was not significant compared with control, suggesting an absence of learning in this phase.

These data demonstrate two points. First, they confirm that strong stimuli can elicit sensitization, whereas weak stimuli do not. Second, they show

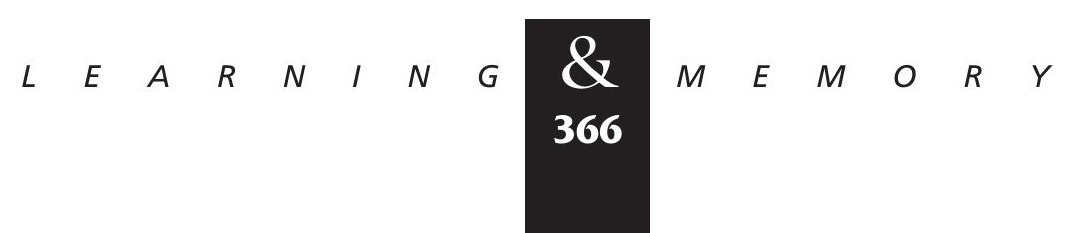




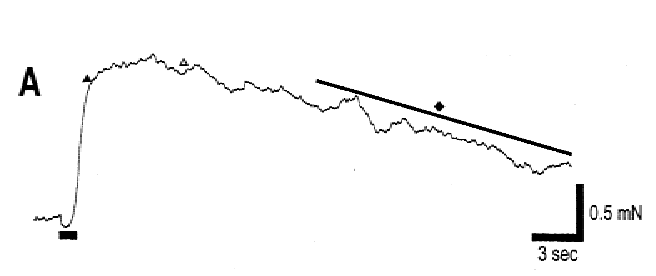

Weak Stimulation
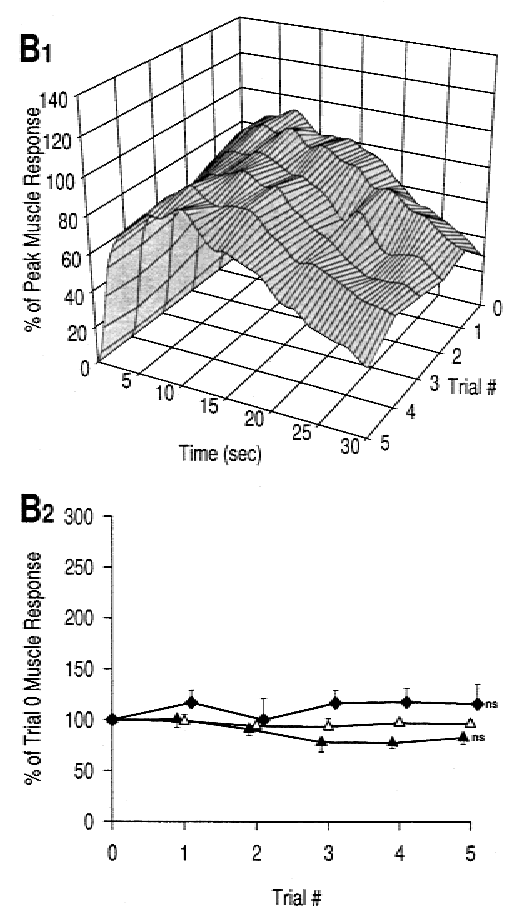

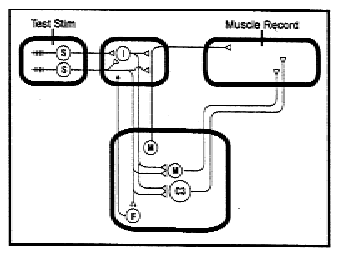

Strong Stimulation
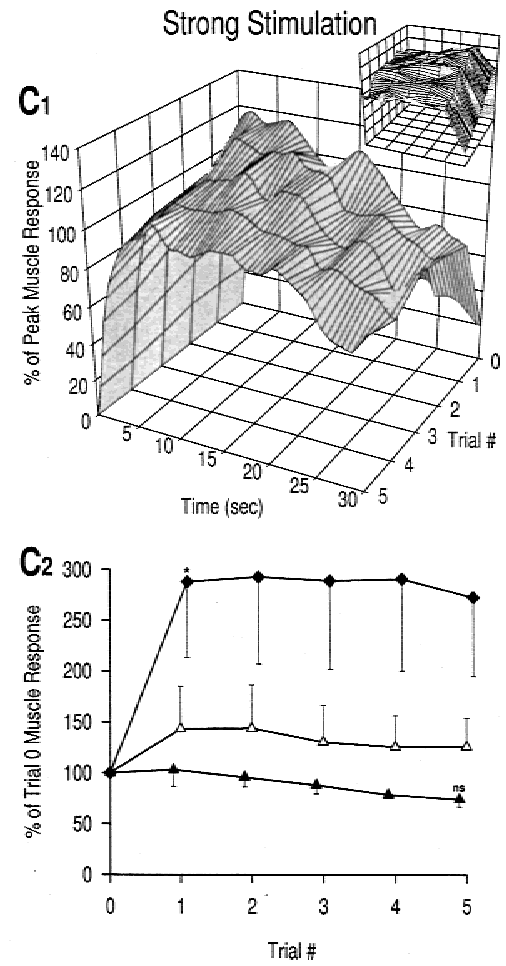

Figure 2: Plasticity of muscle response at long interstimulus intervals. Trial stimuli are applied at 12-min interstimulus intervals; no intervening training stimulation is given. The box in this and subsequent figures shows a circuit diagram with sites of stimulation and recording indicated. (A) Example of muscle response to weak mechanical stimulation in naive preparation. The duration of stimulation is marked by a thick bar at bottom, left of the trace. The three different phases of the response to be measured are also marked: $(\boldsymbol{\Lambda})$ rising phase, response at $2 \mathrm{sec} ;(\triangle)$ peak phase, response at $7 \mathrm{sec}$; $($ ) late phase, response over the last $15 \mathrm{sec}$ (B1) Response profiles, weak stimulation; $n=5$ preparations. (B2) Learning curves, weak stimulation. Error bars in this figure and all other figures represent S.E. The late phase response increases slightly, whereas the rising phase decreases slightly; none of these changes is significant compared with baseline (trial 0) (unpaired $t$-tests with Bonferroni correction for five repeated tests for each phase). These data are consistent with an absence of plasticity; this training condition (weak, infrequent stimulation) is therefore referred to as the control condition. (C1) Response profiles, strong stimulation; $n=3$ preparations. Small graph shows a $90^{\circ}$ clockwise rotated view of the large graph. (C2) Learn-
by trial 1 compared with the control data ing curves, strong stimulation. The late phase response is significantly increased by trial 1 compared with the control data in $B 2$ [asterisk $\left(^{*}\right) P<0.05$; unpaired $t$-test]. The rising phase response did not change significantly between trials 1 and 5 compared with control data [(ns) not significant; unpaired $t$-tests with Bonferroni correction for five tests]. The peak phase showed intermediate changes. These data indicate that habituation does not result from low-frequency stimulation but that sustained sensitization can be induced by strong, infrequent stimulation.

the selective effects of sensitization on the late phase response and therein suggest that facilitation is restricted to the polysynaptic pathway. As predicted, there is no indication of habituation with weak, infrequent stimulation. Given the absence of habituation, it is noteworthy that the effects of sensitization are maintained between trials 1 and 5 in the late phase response (Fig. 2C).

\section{PLASTICITY OF MUSCLE RESPONSE WITH CNS INTACT}

The goal of the next experiment was to induce habituation by increasing the stimulation frequency. With weak stimulation at 2-min intervals, all phases of the muscle response showed the ex- ponential decrease (Fig. 3A) characteristic of habituation (Thompson and Spencer 1966). Reductions in the rising phase and late phase responses were both significant by trial $1(P<0.05)$.

As shown above, repetitive stimulation (i.e., high-frequency) tends to cause habituation, whereas strong stimulation tends to cause sensitization. High-frequency, strong stimulation should simultaneously cause habituation and sensitization. The effects of this sort of stimulation are shown in Figure 3B. The rising phase habituated but at a slower rate than with repetitive weak stimulation; the decrease was significant by trial $2(P<0.05)$. Habituation of the peak phase was also reduced such that there was virtually no change in the response compared with baseline. Plasticity in the

$$
\begin{array}{lllllllllllllll}
L & E & A & R & N & I & N & G & \underset{367}{\mathbf{Z}} & M & E & M & O & R & Y
\end{array}
$$




\section{Prescott and Chase}

Figure 3: Plasticity of muscle response with CNS intact. ( $\mathbf{\Delta})$ Rising phase, response at $2 \mathrm{sec} ;(\triangle)$ peak phase, response at $7 \mathrm{sec}$; $($ ) late phase, response over the last $15 \mathrm{sec}$. (A1) Response profiles, weak stimulation; $n=4$ preparations. (A2) Learning curves, weak stimulation. All phases of the muscle response show an exponential decrease, though at slightly different rates, as is standard for habituation. Reductions in the rising phase and late phase are both significant by trial 1 [asterisk $\left(^{*}\right) P<0.05$; unpaired $t$-tests]. (B1) Response profiles, strong stimulation; $n=5$ preparations. Small graph shows a $90^{\circ}$ clockwise rotated view of the large graph. (B2) Learning curves, strong stimulation. Different phases of the muscle response exhibit very different changes. The rising phase still exhibits habituation though at a reduced rate compared with $A 2$; the reduction is significant by trial 2 [asterisk (*), $P<0.05$; unpaired $t$-tests with Bonferroni correction for two tests]. In contrast, the late phase transiently sensitizes; the increase is not significant compared with control but is significant compared with the equivalent point on $A$ [ asterisk (*), $P<0.05$; unpaired $t$-test]. Changes in the peak phase are again intermediate compared with changes in the other two phases. The nonmonotonic changes (as seen in the late phase response) are a defining feature of dual-process learning. These data indicate that habituation affects all phases of the response, whereas sensitization predominantly affects the late phase.

late phase was the most profoundly altered with the response transiently rising above baseline for trials $1-3$. The response reached $182.3 \pm 40.7 \%$ in trial 1, which, though not significantly larger than trial 1 of the control condition, was significantly larger than trial 1 in Figure $3 \mathrm{~A}(P<0.05)$. It is very important to notice the decrease in the late phase response between trials 1 and 5 (Fig. 3B). The nonmonotonic changes in late phase response amplitude are consistent with dual-process learning and suggest that habituation and sensitization occur simultaneously. This is in striking contrast to the learning kinetics for the late phase response in Figure 2C wherein only sensitization is evident.

The rising phase of the muscle response is mediated monosynaptically, whereas the late phase is mediated polysynaptically (see Introduction). The findings indicate that, with weak stimulation, ha-

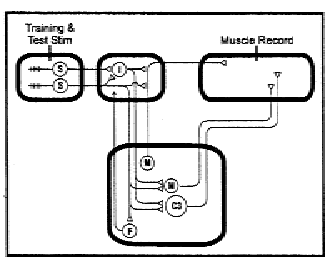

Weak Stimulation
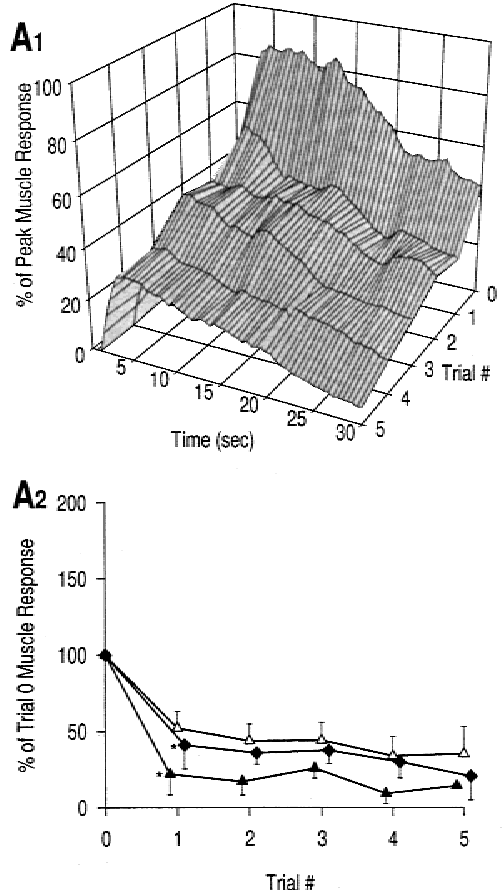

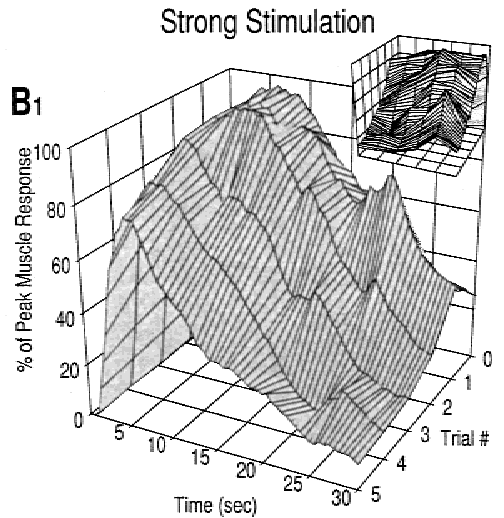

B2

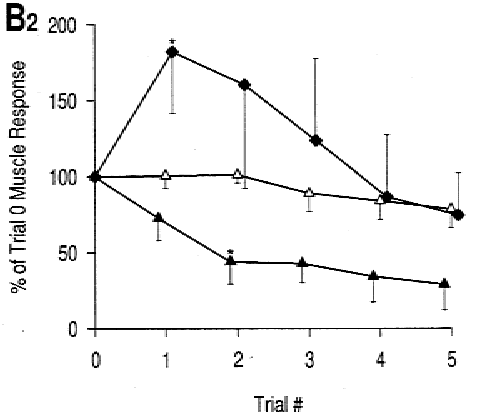

bituation affects all phases of the muscle response, suggesting that depression is expressed in both monosynaptic and polysynaptic pathways. With strong stimulation, only the late phase shows clear evidence of sensitization, suggesting that facilitation occurs selectively in the polysynaptic pathway. Reduction in the rate of habituation of the rising phase response with strong stimulation compared with weak stimulation does not indicate sensitization because habituation occurs more slowly with higher stimulus intensities without any contribution from sensitization (Thompson and Spencer 1966; see below); notably, the learning curve for this phase still displays a monotonic exponential decrease. The peak phase is presumably mediated jointly through both monosynaptic and polysynaptic pathways, which would explain its intermediate plasticity.

$$
\begin{array}{lllllllllllllll}
\hline & E & A & R & N & I & N & G & \underset{368}{\mathbf{Z}} & M & E & M & O & R & Y
\end{array}
$$


STATISTICAL ANALYSIS OF EFFECTS OF STIMULUS PARAMETERS

Two-way ANOVAs were used to further investigate the effects of stimulus intensity and frequency on plasticity of the rising phase and late phase of the muscle response. Comparing the late phase responses in trial 1 for different stimulation parameters (data from Figs. 2 and 3), a two-way ANOVA showed that intensity had a significant effect $\left(F_{\text {intensity }}=10.00, P<0.01, d f=1\right)$. Frequency had an effect that fell just short of significance after allowing for the effects of intensity $\left(F_{\text {frequency }}=4.28, P=0.06, d f=1\right)$. However, a two-way ANOVA on the late phase response in trial 5 showed that frequency had a significant effect on plasticity after allowing for differences in intensity $\quad\left(F_{\text {frequency }}=10.06, \quad P<0.01, \quad d f=1\right)$, whereas intensity no longer had a significant effect $\left(F_{\text {intensity }}=2.67, P>0.05, d f=1\right)$. Repeating the ANOVA on trials 2-4 showed that intensity had a significant effect in trial $2(P<0.05)$, but the effect was not significant in later trials. In contrast, the effects of frequency only became significant at trial $3(P<0.05)$ and increased in later trials. The fact that stimulus frequency and intensity both affect plasticity of the late phase response is further evidence that both habituation and sensitization compete to determine net changes in this phase of the response and, moreover, that the balance between the two learning processes shifts in favor of habituation as training progresses.

Analysis of the rising phase response showed a different pattern of effects. Two-way ANOVAs on trials 1-5 showed that stimulus intensity did not have a significant effect on plasticity in any of the trials. Stimulus frequency, on the other hand, had a significant effect in all trials (at least $P<0.05$ ). These results are consistent with the argument that the rising phase of the response is affected by habituation but not by sensitization.

\section{PLASTICITY OF MUSCLE RESPONSE AFTER CNS LESION}

Previous studies in Helix have shown that the rate, amplitude, and duration of muscle contraction are reduced after a CNS lesion (Prescott and Chase 1996; Prescott et al. 1997), but the response is still quite robust and can exhibit the effects of learning. Unlike habituation that does not require the CNS, sensitization requires the $\mathrm{CNS}$ for induction but not for expression (Prescott and Chase
1996). Therefore, preparations with CNS lesions were trained with either weak or strong stimuli for comparison to the plasticity in preparations with the CNS intact. The prediction was that habituation would be intact, whereas sensitization would be absent in those preparations with a CNS lesion.

As when the CNS is intact, repeated weak stimulation after a CNS lesion resulted in habituation of all phases of the muscle response (Fig. 4A). Reductions in the rising phase and in the peak phase were both significant by trial $1(P<0.05$ and $P<0.01$, respectively). Repeated strong stimulation with a CNS lesion, on the other hand, did not elicit the same plasticity as with an intact CNS. Instead, all phases of the response habituated with standard kinetics (Fig. 4B); reduction in the rising phase was significant by trial $1(P<0.05)$, and reduction in the late phase was significant by trial 4 $(P<0.01)$. Furthermore, kinetics of plasticity in the late phase were qualitatively changed in that there was no transient increase in the response above baseline as seen when the CNS was intact (Fig. 3B). These changes are attributable to a loss of facilitation caused by the CNS lesion, which is consistent with the necessity of the CNS for sensitization's induction (see above). Habituation of the rising phase is virtually unchanged from that seen in Figure 3B, supporting the previous argument that facilitation does not occur in the monosynaptic pathway.

As mentioned above, habituation is reduced when stimulus intensity is increased (Thompson and Spencer 1966). This is an intrinsic property of habituation and does not rely on the incremental effects of sensitization. The reduction of habituation in the absence of sensitization is clearly seen by comparing the plasticity elicited by weak (Fig. 4A) and strong (Fig. 4B) stimulation after a CNS lesion. The argument for reduced habituation rather than occult sensitization is supported by the fact that the decrease is monotonic, rather than showing any transient increase suggestive of sensitization.

\section{PLASTICITY OF OLFACTORY NERVE RESPONSE AFTER CNS LESION}

Having investigated the learning kinetics of tentacle withdrawal, the next step was to explore the underlying network plasticity. We began by looking at plasticity in the earliest (most upstream) part of the circuit and then moved progressively downstream. The numbered loci in Figure 1 indi-

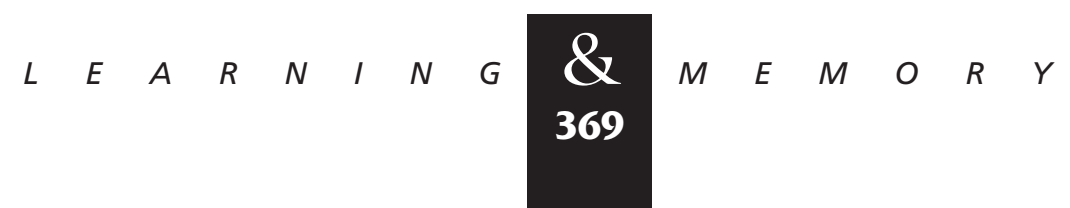




\section{Prescott and Chase}

Figure 4: Plasticity of muscle response after CNS lesion. ( $\Delta$ ) Rising phase, response at $2 \mathrm{sec} ;(\triangle)$ peak phase, re-

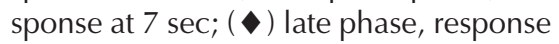
over last 15 sec. (A1) Response profiles, weak stimulation; $n=5$ preparations. (A2) Learning curves, weak stimulation. As with the CNS intact, weak stimulation results in habituation of all phases of the response. Reductions in the rising phase and late phase are both significant by trial 1 [asterisk $(*), P<0.05$; asterisks $(* *), P<0.01$; unpaired $t$-tests]. (B1) Response profiles, strong stimulation; $n=3$ preparations. (B2) Learning curves, strong stimulation. Reduction in the rising phase is still significant at trial 1 [asterisk $\left(^{*}\right) \quad P<0.05$; unpaired $t$-test), whereas the reduction in the peak phase reaches significance at trial 4 [asterisk (*), $P<0.05$; unpaired $t$-tests with Bonferroni correction for four tests]. These curves are markedly different from the equivalent curves in Fig. 3B2, that is, there is no transient increase above baseline. This confirms that the CNS is necessary for the induction of sensitization, which also reflects the heterosynaptic nature of facilitation. Comparison of $A$ and $B$ shows that even in the absence of sensitization, the rate and degree of habituation are less under conditions of strong stimulation compared with weak stimulation, consistent with the standard kinetics of habituation.

cate possible sites of plasticity and generally follow the chronology of our investigation.

To investigate plasticity in the upstream circuit, the CNS was lesioned and the distal stump of the olfactory nerve was taken up in a suction electrode to record afferent neural activity. Figure 5A shows a sample recording from the olfactory nerve. Response profiles are shown only for strong stimulation (Fig. 5B1); data from weak stimulation are very similar. Learning curves for both stimulus strengths are shown in Figure 5B2. The peak phase, occurring very early in the response (1.25 sec; see Fig. 5A), was not significantly different from the baseline response at any trial, for both weak and strong stimulation. Given the short latency to the peak phase, the majority of this response constitutes activity in the monosynaptic pathway because activity in the polysynaptic pathway is delayed by an upstream synapse and, more-
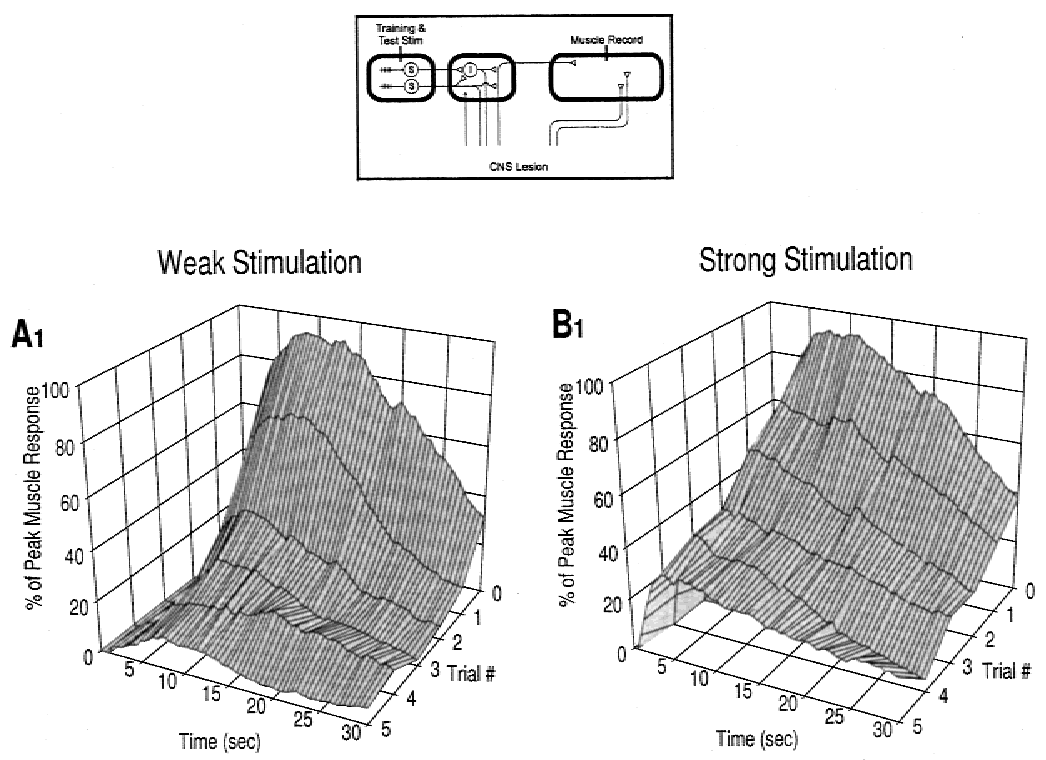
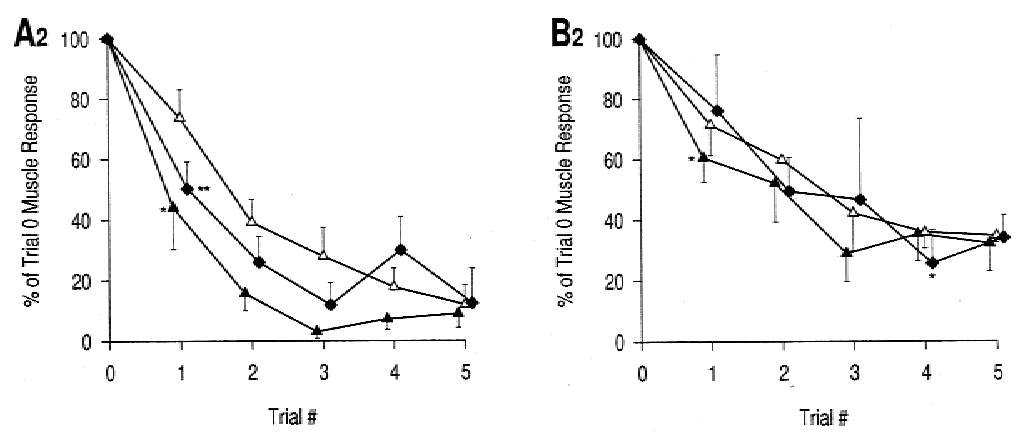

Weak Stimulation over, never seems to reach such a high firing frequency. The absence of plasticity in the peak phase is therefore consistent with a direct projection of sensory neurons to the CNS (see Fig. 1), meaning that there is no plastic synapse upstream of the recording site nor that decreased neuronal excitability plays any role in depression. The short duration of the nonplastic phase (see Fig. 5B1) reflects the phasic nature of activity in the monosynaptic pathway.

The prolonged neural activity transmitted through the polysynaptic pathway (i.e., late phase), on the other hand, showed a progressive decrease with training. This occurred for both strong and weak stimulation, as expected with a CNS lesion (because there is no sensitization). The reduction compared baseline was significant by trial 1 for both stimulus strengths $(P<0.05)$. The most likely explanation for this reduction is depres-

$$
\begin{array}{lllllllllllllll}
L & E & A & R & N & I & N & G & \underset{370}{\mathbf{Z}} & M & E & M & O & R & Y
\end{array}
$$



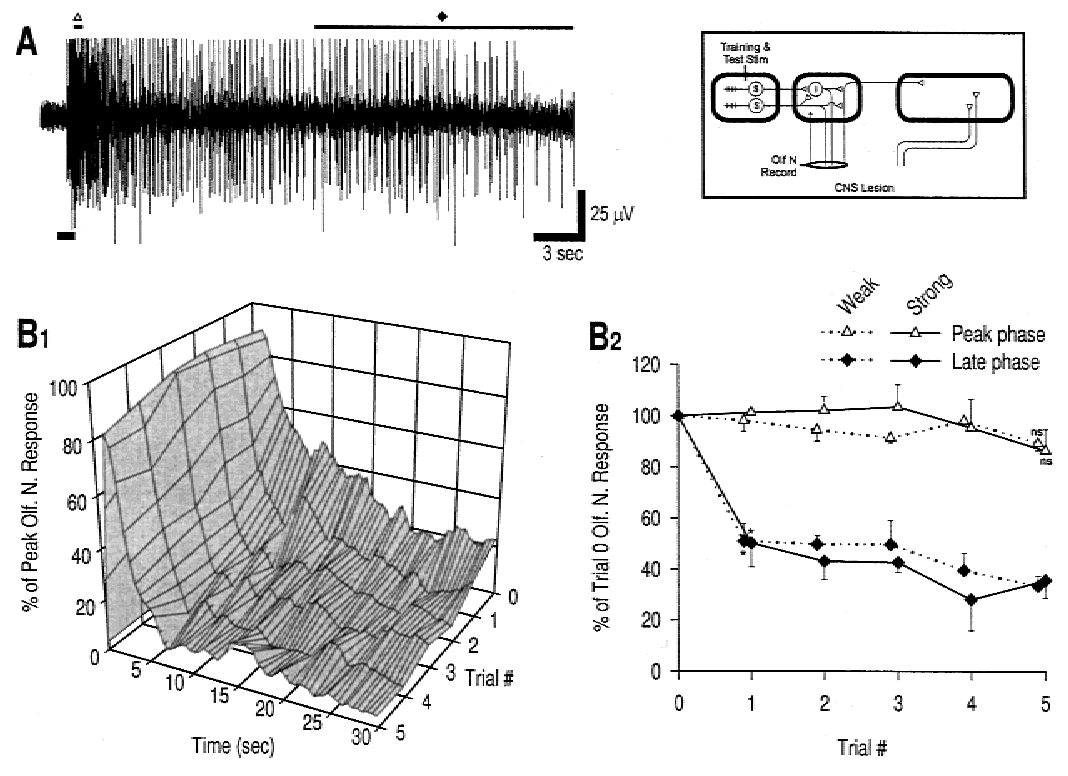

Figure 5: Plasticity of olfactory nerve response after CNS lesion. (A) Example of olfactory nerve response to weak mechanical stimulation in a naive preparation. The tops of the action potentials were truncated during recording. The duration of stimulation is marked by a thick bar at bottom, left of the trace. The two different phases of the response to be measured are also marked: $(\triangle)$ peak phase, response at $1.25 \mathrm{sec} ;(\diamond)$ late phase, response over the last 15 sec. (B1) Response profiles, strong stimulation; $n=3$ preparations. Response profiles for weak stimulation (not shown, $n=3$ preparations) are very similar to those for strong stimulation. (B2) Learning curves, weak and strong stimulation. Consistent with the findings in Fig. 4, facilitation does not occur in the absence of the CNS. The peak phase shows no significant change from baseline for either weak or strong stimulation [(ns) not significant; unpaired t-tests with Bonferroni correction for five tests]. In contrast, prolonged neural activity of the late phase is significantly reduced compared with baseline by trial 1 for both stimulus strengths [asterisk $\left(^{*}\right), P<0.05$; unpaired $t$-tests]. This pattern of depression is consistent with plasticity at locus 1.

sion at the synapses between sensory neurons and interneurons (locus 1 on Fig. 1) because prolonged firing occurs in the interneurons.

\section{PLASTICITY OF C3 RESPONSE WITH CNS INTACT}

$\mathrm{C} 3$ is a giant motoneuron that contributes substantially to the mediation of tentacle withdrawal (Prescott et al. 1997). Its large size allows for relatively good intracellular recording, which also provides the benefit of selective, intracellular stimulation. The convergent input onto $\mathrm{C} 3$ from both sensory neurons and interneurons (Fig. 1) is reflected in its response, which consists of a high-frequency burst followed by more prolonged, tonic activity (Fig. 6A).

Unlike the olfactory nerve response, the C3 response showed a decrease in its peak phase over the course of training for both weak and strong stimulation (Fig. 6B,C). The responses were consistently lower than baseline for both stimulus strengths, but the reduction did not reach significance in either case. Given the very high firing frequency in the peak phase, decreased firing may not accurately reflect decreased synaptic input to C3 because of response saturation, which could explain the attenuated decrement in this phase (Fig. 6B,C). As expected, the decrease was slower in the case of strong stimulation. The $\mathrm{C} 3$ response decrement is probably mediated through depression at the synapse between sensory neurons and C3 (locus 2).

With weak stimulation, the late phase $\mathrm{C} 3$ response also decreased (Fig. 6B); the reduction was significant by trial $1(P<0.05)$. This could be due to depression at locus 1 and/or at locus 3. Using the simplifying assumption of linear additivity to subtract the effects of depression at locus 1 (see figure legend for details), Figure $7 \mathrm{~A}$ demonstrates that the reduction in the late phase olfactory nerve response is sufficient to account for reduction in the late phase $\mathrm{C} 3$ response. This suggests that depression occurs upstream at locus 1 rather than farther downstream at locus 3.

Changes in the late phase response were qualitatively different depending on whether training was with weak or strong stimulation (Fig. 6, cf. B and $\mathrm{C}$ ). After training with strong stimulation, the late phase $\mathrm{C} 3$ response showed a transient increase (Fig. 6C) reminiscent of plasticity in the late phase muscle response with the CNS intact (Fig. 3B). The response at trial 1 was not, however, significantly increased compared with baseline or with Figure 6B. However, a one-way ANOVA on the late phase response using data from trials 1-5 showed that stimulus intensity did have a significant effect

$$
\begin{array}{lllllllllllllll}
L & E & A & R & N & I & N & G & \begin{array}{l}
\boldsymbol{Q} \\
\mathbf{3 7 1}
\end{array} & M & E & M & O & R & Y
\end{array}
$$




\section{Prescott and Chase}

Figure 6: Plasticity of $\mathrm{C} 3$ response. (A) Example of $\mathrm{C} 3$ response to weak mechanical stimulation in a naive preparation. The duration of stimulation is marked by a thick bar at bottom, left of the trace. The two different phases of the response to be measured are also marked: $(\triangle)$ peak phase, response at $1.75 \mathrm{sec}$; $\diamond$ ) late phase, response over the last $15 \mathrm{sec}$. This example shows a relatively robust late phase response. (B1) Response profiles, weak stimulation; $n=3$ preparations. (B2) Learning curves, weak stimulation. The peak phase response decreases between trials 0 and 5, but the reduction does not reach significance compared with baseline [(ns) not significant; unpaired $t$-tests with Bonferroni correction for five tests]; however, response saturation may attenuate the observable decrease in spike number (see Results). Depression at locus 2 is the most probable cause for the decrease in the peak phase $\mathrm{C} 3$ response. Reduction in the late phase compared with baseline is significant by trial 1 [asterisk $(*), P<0.05$; unpaired $t$-test]. Depression at locus 1 is the most probable cause for this decrease (see Results). (C1) Response profiles, strong stimulation; $n=3$ preparations. (C2) Learning curves, strong stimulation. Reduction of
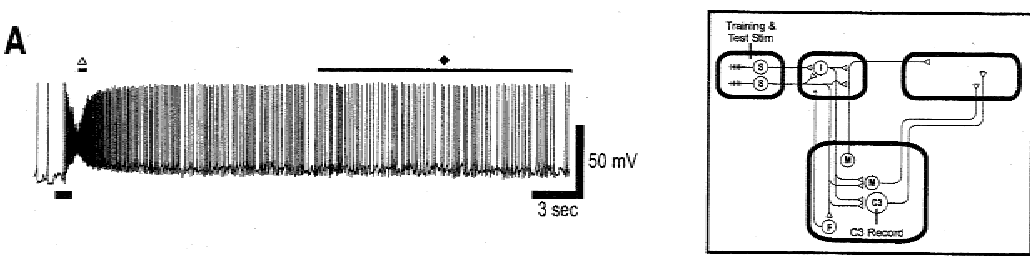

Weak Stimulation
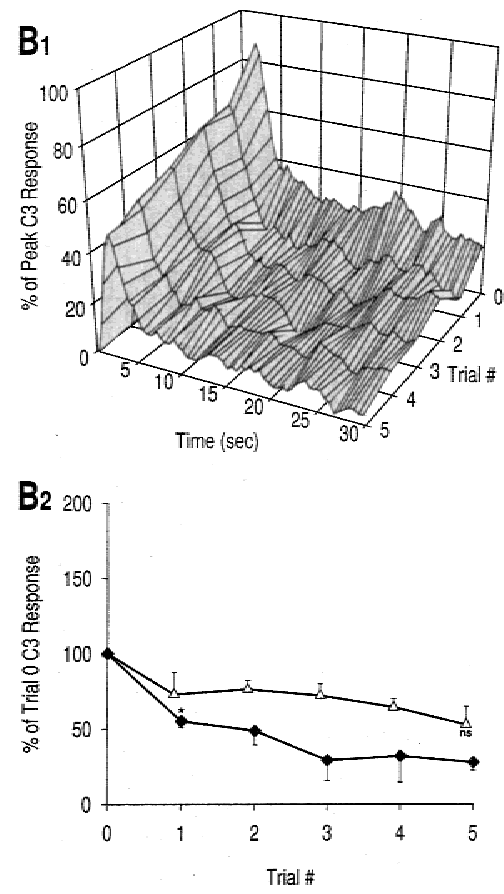

Strong Stimulation
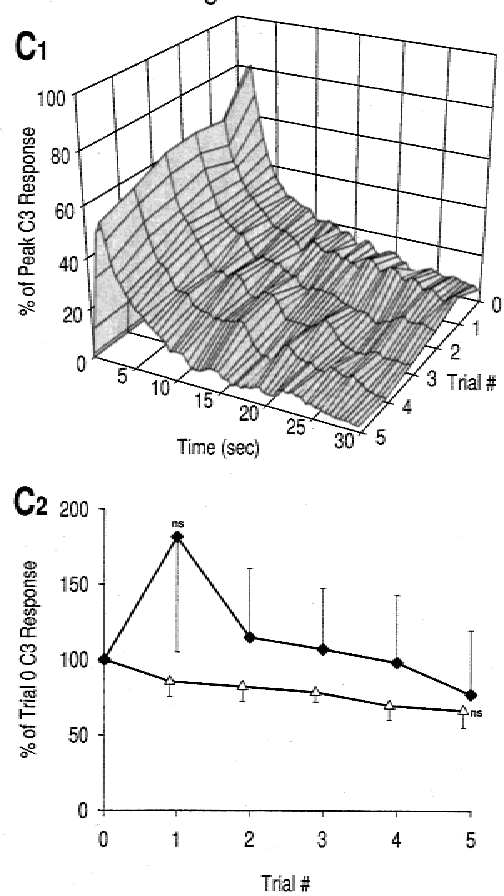

the peak phase is less than with weak

stimulation [the response is not significantly altered from baseline; (ns) not significant; unpaired t-tests with Bonferroni correction for five tests], but the kinetics are otherwise quite similar with the response showing a progressive decrement. In contrast, the late phase of the C3 response shows a transient increase very similar to that seen in the late phase muscle response (Fig. 3D). The increase at trial 1 is not significant compared with baseline or to data in B2 [(ns) not significant; unpaired $t$-tests]; however, results of a one-way ANOVA described in the text suggest that sensitization does affect the late phase response. These data are consistent with selective expression of facilitation in the polysynaptic pathway.

$\left(F_{\text {intensity }}=7.58, P<0.05, d f=1\right)$ that suggests that sensitization affects the late phase. Despite the variability between preparations, plasticity in the late phase response seen after strong stimulation appears quite different from that seen after weak stimulation.

The above data provide evidence for facilitation in at least one component of the central polysynaptic pathway. Past experiments in which the CNS was lesioned after induction of sensitization suggest, in fact, that the majority of facilitation is probably expressed in the peripheral pathways (Prescott and Chase 1996). Facilitation in peripheral pathways cannot be directly recorded for technical reasons, but data reported below confirm that such facilitation does occur and appears to be much more robust than in the central pathway. Common expression of facilitation between central and peripheral pathways could be explained by facilitation at locus 1 and/or facilitation at both loci 3 and 5. Facilitation at the neuromuscular junction (loci 6 and 7) is also possible.

Another issue to consider for localizing facilitation is the relationship between facilitation and prolongation and their respective sites of expression. Prolongation is the phenomenon wherein the excitation caused by a brief stimulus is transformed into a prolonged neuronal discharge. Previous experiments have shown that the transformation occurs in the tentacle ganglion, most likely at locus 1 , and that the degree of prolongation is proportional to the signal intensity (Prescott et al. 1997). On the 
A

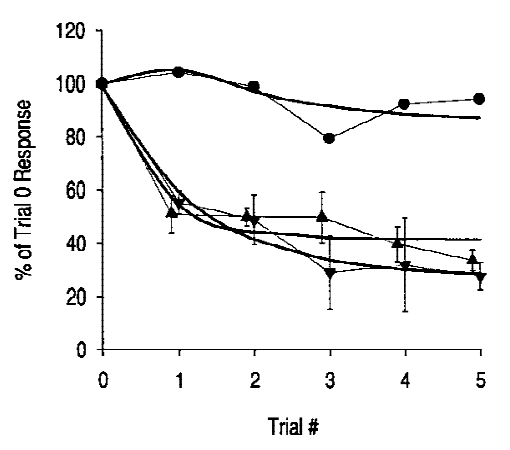

B

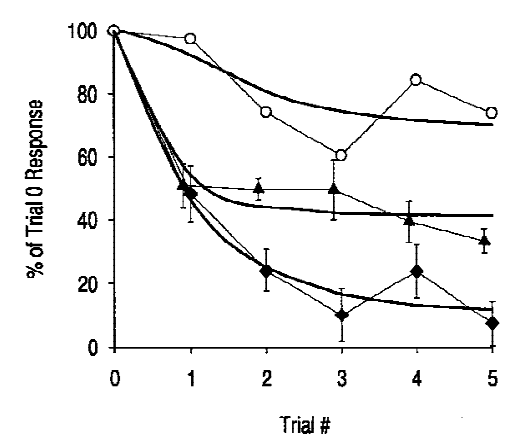

Figure 7: Localization of depression. (A) Depression in the central pathway, weak stimulation. Original data for late phase responses have been fit with single exponential curves. Reduction of the late phase olfactory nerve response indicates depression at locus 1 (late phase data from Fig. 5B2, $\mathbf{\Delta}$ ), whereas reduction of the late phase $\mathrm{C} 3$ response indicates the summed depression at loci 1 and 3 (late phase data from Fig. 6B2, $\mathbf{\nabla}$ ). Depression at locus $3(-)$ was calculated by subtracting depression at locus 1 from depression at loci $1+3$, using the simplifying assumption of linear additivity. Inferred plasticity at locus 3 . The results indicate that depression at locus 3 contributes little to total depression. $(B)$ Depression in the peripheral pathway, weak stimulation. The same strategy is used as in $A$ to subtract depression at locus 1 (late phase data from Fig. 5B2, $\mathbf{\Delta}$ ) from depression at loci $1+5+7$ (late phase data from Fig. 4A2, $\bullet$ ) to show that depression at locus 5 and/or 7 makes only a minor contribution compared with depression at locus 1. ( $\bigcirc)$ Inferred plasticity at loci 5 and 7 . Together with the findings of Figs. 4 and 5, these data indicate that decrement of sensory neuron output is the main cause of decreased transmission through the neural circuit.

basis of the influence of signal intensity, facilitation causing increased transmission at locus 1 would be expected to enhance prolongation, whereas facilitation downstream would likely fail to have any such effect. It seems highly plausible, therefore, that facilitation conferring sensitization in the late phase response occurs at locus 1 , but this does not exclude plasticity farther downstream in either the central or peripheral polysynaptic pathways.

\section{PLASTICITY AT OTHER SITES IN THE CIRCUIT}

Having investigated plasticity in the central pathway, the next goal was to understand plasticity at analogous sites in the peripheral pathway. Although direct measurement of plasticity in this pathway is complicated by technical difficulties, data already presented allow one to infer that reduction of the neural signal in the peripheral pathway (reflected by decreased muscle response after CNS lesion; see Fig. 4) is mostly attributable to depression at an early locus. For habituation of the early phase, depression probably occurs at the peripheral synapse between sensory neurons and motoneurons (locus 4). Using the same method of subtraction as in Figure $7 \mathrm{~A}$, Figure $7 \mathrm{~B}$ shows that the majority of reduction in the late phase muscle response is most likely attributable to depression at locus 1 . In both cases, the localization of depression is in keeping with previous data (see above) indicating that the output synapses of sensory neurons are prone to depression. There may also be plasticity farther downstream, such as depression at the neuromuscular junction (locus 7), but any such contribution is probably minor.

We examined the question of neuromuscular plasticity mediated through the central pathway by directly exciting C3 by injection of depolarizing current ( 1.0 nA over $8 \mathrm{sec})$ to cause spiking equivalent in intensity to that caused by weak mechanical stimulation of the olfactory epithelium. This intracellular stimulation was repeated using the same training schedule as in other experiments; total spike number and peak muscle response were measured. Between trials 0 and 5, the C3 response decreased by only 3.3\%, whereas the muscle response decreased by only $4.0 \%(n=3$ preparations). Repeated stimulation therefore caused neither reduction in C3's excitability nor depression of C3's output (i.e., at locus 6).

The possibility of post-tetanic potentiation at C3's neuromuscular junction (locus 6) was also tested. Various intensities, durations, and combinations of stimulation were tried, but in no instance was there evidence of increased synaptic transmission to the muscle. Results also suggests that C3 is not responsible for inducing heterosynaptic facilitation. The possibility of heterosynaptic facilitation at locus 6 under other training conditions is not

$$
\begin{array}{lllllllllllllll}
L & E & A & R & N & I & N & G & \underset{373}{\mathbf{Z}} & M & E & M & O & R & Y
\end{array}
$$




\section{Prescott and Chase}

ruled out by these experiments, but it seems unlikely given other results. All available data suggest that locus 6 is nonplastic. Separate stimulation experiments suggest that post-tetanic potentiation is also absent from other neuromuscular junctions (see below).

\section{INDUCTION OF SENSITIZATION \\ BY ELECTRICAL STIMULATION}

Given the necessity of the CNS for the induction of sensitization, we hypothesized that facilitatory neurons project from the CNS to the periphery where they effect an increase in synaptic transmission selectively in the polysynaptic pathway (see Fig. 1). Given the upstream location of depression relative to facilitatory neurons, activation of facilitatory neurons would tend to wane as depression develops, thereby causing a reduction in facilitation. This has important implications for dualprocess learning (see Discussion).

Because facilitatory neurons have not yet been identified in this system, the scenario described above cannot be directly demonstrated. As an alternative, the olfactory nerve was lesioned and electrically stimulated at its distal end, first, to show that facilitation can be expressed in the periphery as suggested earlier in the text, second, to show that this stimulation can elicit sensitization (therein supporting the existence of centrifugal facilitatory neurons), and third, to investigate the kinetics of sensitization elicited in such a manner. The stimulus used for each training trial consisted of 50 pulses delivered at $10 \mathrm{~Hz}$, each pulse being $20 \mathrm{msec}$ in duration and $500 \mathrm{mV}$ in intensity; weak mechanical stimulation was used for the test trials.

Direct stimulation of the olfactory nerve elicited sensitization (Fig. 8). The rising phase response, although increased, was not significantly changed in any trial compared with control data. On the other hand, the increase in the late phase response was significant by trial $1(P<0.01)$. Because the CNS had been lesioned, these results suggest that the axons of central facilitatory neurons were directly excited, causing facilitation in the periphery. The effects of sensitization were similar to those observed in response to mechanical stimulation (Figs. 2 and 3); specifically, the late phase showed a large increase, whereas the other two phases showed only small increases. One could argue that this sensitization was possibly caused by post-tetanic potentiation rather than by heterosynaptic facilitation, but such an argument is not supported by the differential effects of sensitization depending on response phase.

An important point to notice in Figure 8 is the kinetics of the sensitization. Although there is a slight decrease after the largest increase (at trial 1)
Figure 8: Plasticity of muscle response with olfactory nerve stimulation. Electrical stimulation was applied to the distal end of the cut olfactory nerve for training; weak mechanical stimulation was used for testing. ( $\mathbf{\Delta})$ Rising phase, response at 2 sec; $(\triangle)$ peak phase, response at $7 \mathrm{sec} ;(\bullet)$ late phase, response over the last $15 \mathrm{sec}$. (A) Response profiles; $n=3$ preparations. The small graph shows a $90^{\circ}$ clockwise rotated view of the large graph. (B) Learning curves. The rising phase increased above baseline, but the response is not significantly larger than control data at any trial [(ns) not significant; unpaired $t$ tests with Bonferroni correction for five tests]. The late phase response showed the greatest increase and is significantly larger than control data by trial 1 [asterisks $(* *)$, $P<0.01$; unpaired $t$-test). This pattern of effects in which the late phase is greatly increased whereas the other phases are less affected is similar to the effects observed with training by mechanical stimulation (Figs. 2 and 3). These data therefore support the argument that facilitatory neurons project from the cerebral ganglia (as shown in Fig. 1) and effect facilitation peripherally in the polysynaptic pathways.
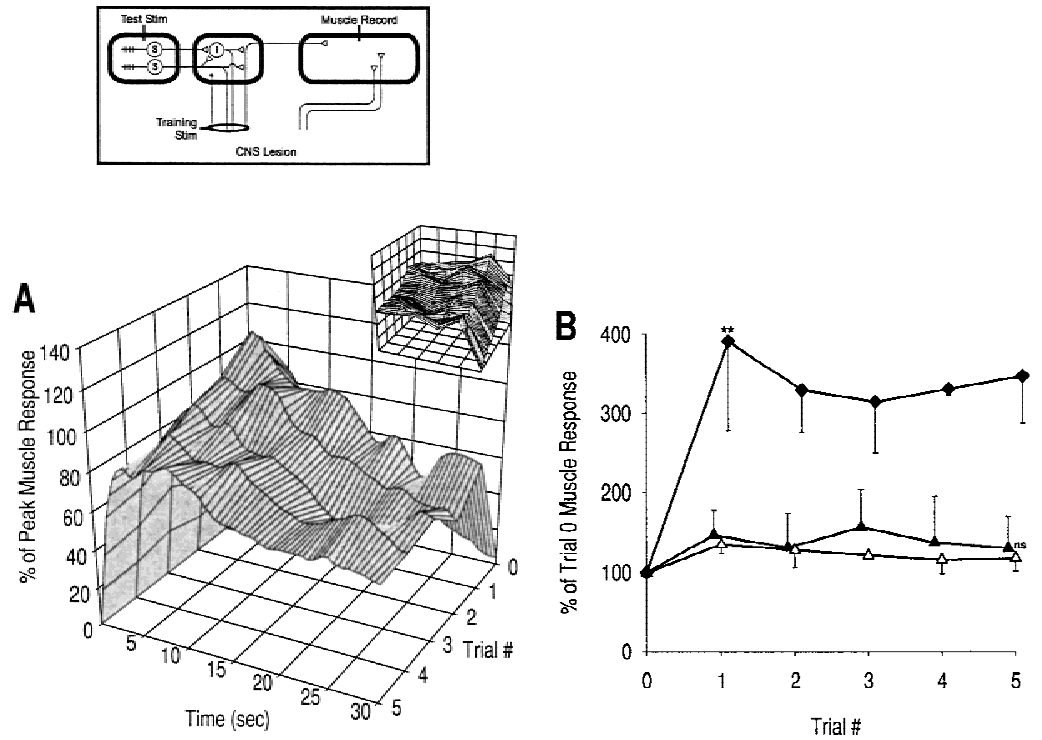

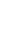


in the late phase response, the increase is maintained well above baseline. In this regard, the kinetics of plasticity in the late phase response are more similar to those observed in Figure $2 \mathrm{C}$ than to those in Figure 3B; furthermore, there is no evidence of decreases in the other phases of the response. These results suggest that repeated stimulation of the olfactory nerve, although causing sensitization through direct excitation of the facilitatory neurons, did not elicit habituation because the site of stimulation was downstream of the locus of depression.

\section{Discussion}

Careful analysis of the tentacle withdrawal reflex in Helix shows that different phases of the response can change independently through learning and are differentially influenced by habituation and sensitization. Previous data from this system and from Aplysia (see Introduction) suggest that the early phase of withdrawal responses is mediated by monosynaptic neural pathways. Data from Figure 6 show that the monosynaptic pathway displays depression, which is consistent with habituation of the rising phase muscle response seen in Figures 3 and 4 . The polysynaptic pathway, which is believed to be important for the later part of the response by virtue of its prolonged activity, also expresses depression (Figs. 5 and 6), the effects of which are reflected in the late phase muscle response (Figs. 3 and 4). But whereas depression is common to both pathways, facilitation is selectively expressed in the polysynaptic pathway as suggested by the dissociation of plasticity in the early and late phase responses in both C3 (Fig. 6) and the muscle (Figs. 2, 3, and 8). These results are consistent with our previous report of the differential effects of habituation and sensitization on response amplitude and duration (Prescott and Chase 1996). Recent experiments in Tritonia (Brown et al. 1996) and in Aplysia (Hawkins et al. 1998) have also demonstrated dissociative changes in different components of behavior, leading the authors to postulate that plasticity at different loci in the network underlies changes in different components of the behavior.

Table 1 summarizes the localization of plasticity within the neural network mediating tentacle withdrawal in Helix. The vast majority of plasticity occurs at the output synapses of sensory neurons (loci 1, 2, and 4), but, as noted above, net plasticity is not the same between monosynaptic and poly-
Table 1: Summary of plastic loci

\begin{tabular}{lccc}
\hline & $\begin{array}{c}\text { Number } \\
\text { on }\end{array}$ & & \\
Synapse & Fig. 1 & Depression & Facilitation \\
\hline S-I & 1 & yes & yes \\
S-C3 & 2 & yes & no \\
I-C3 & 3 & no & no \\
S-M & 4 & yes & no \\
I-M & 5 & no & ?) \\
C3-muscle & 6 & no & no \\
M-muscle & 7 & no & (?) \\
\hline
\end{tabular}

(C3) Identified motoneuron; (F) facilitatory neurons; (I) interneurons; $(\mathrm{M})$ motoneurons additional to $\mathrm{C} 3$; (S) sensory neurons.

synaptic pathways, with facilitation selectively expressed in the latter (most likely at locus 1 ). The balance between the opposing forms of plasticity is significantly influenced by the intensity and frequency of stimulation. By taking advantage of this sensitivity to stimulation parameters, as well as the necessity of the CNS for sensitization, data were collected to investigate how depression and facilitation interact to determine net plasticity and the kinetics of dual-process learning, as will be explained below.

\section{HABITUATION AND DEPRESSION}

It is widely held that synaptic depression is causally related to behavioral habituation (see Christoffersen 1997) though other mechanisms causing reduced neural transmission have been described, such as decreased sensory neuron excitability (Walters et al. 1983). In Aplysia, homosynaptic depression is very robust at sensory neuron output synapses (Castellucci et al. 1970). Plasticity at an analogous, upstream position seems to occur in many circuits (for reviews, see Menzel and Bicker 1987; Prescott 1998). Data indicate that the tentacle withdrawal reflex of Helix similarly expresses depression at the sensory neuron output synapses (loci 1, 2, and 4).

There are many consequences of depression occurring so early in the circuit. Because depression precedes divergence of monosynaptic and polysynaptic pathways, depression affects transmission through both pathways, as seen in Aplysia (Hawkins et al. 1981). The ubiquitous effects of depression on the rate (rising phase), peak ampli-

$$
\begin{array}{llllllllllllllll}
\hline & E & A & R & N & I & N & G & \boldsymbol{\&} \\
375 & M & E & M & O & R & Y
\end{array}
$$


tude (peak phase), and duration (late phase) of the response seen in this study support the same conclusion. Another consequence of depression so early in the circuit is that input to downstream facilitatory neurons will wane as depression develops, which has important consequences for the kinetics of learning (see below).

\section{SENSITIZATION AND FACILITATION}

Just as depression is thought to cause habituation, facilitation is thought to underlie sensitization. An increase in synaptic transmission can be effected by many different mechanisms (Fisher et al. 1997). By using the word facilitation, we wish only to indicate that transmission through the plastic locus is increased and that the mechanism responsible for this change is heterosynaptic, that is, produced by influences extrinsic to the plastic locus. In the case of tentacle withdrawal, the need for heterosynaptic modulation is illustrated by the necessity of the CNS for facilitation (cf. Figs. 3 and 4), especially given that facilitation can be expressed in the periphery (Fig. 8; Prescott and Chase 1996).

In Aplysia, the mechanism for presynaptic facilitation has been well described (Castellucci and Kandel 1976; for review, see Byrne and Kandel 1996). A similar mechanism probably occurs in $\mathrm{He}$ lix. Facilitation also tends to occur at an upstream position but only at a subset of the synapses that display depression. In other words, depression tends to be cellwide, but facilitation is branch specific depending on where modulatory transmitter is received and where it induces facilitation (Clark and Kandel 1984; Martin et al. 1997). The branch specificity of facilitation is important for explaining the differential effects of sensitization on different components of the behavior as observed in Aplysia (e.g., Stopfer and Carew 1996) and in the current study (Figs. 2, 3, 6, and 8). The most plausible scenario for facilitation in the tentacle withdrawal reflex is that facilitatory neurons projecting from the CNS (Fig. 1) cause facilitation peripherally, in the polysynaptic pathways.

Although the effects of facilitation predominate in the polysynaptic pathway underlying tentacle withdrawal in Helix, precise localization of the facilitated loci is uncertain (Table 1). A large component probably occurs at locus 1 , first, because of facilitation's common expression in central and peripheral polysynaptic pathways and, second, because of the position of locus 1 relative to the site of prolongation. However, both lines of argument are circumstantial, and facilitation's more precise localization warrants further investigation. In Aplysia for instance, facilitation at sensory neuron synapses is well documented, but comparatively recent findings have emphasized plasticity at other sites and by other mechanisms such as a reduction of inhibition (Frost et al. 1988; Fischer and Carew 1993; Trudeau and Castellucci 1993a,b; Cohen et al. 1997). Although inhibition can occur in the circuit mediating tentacle withdrawal, modulation of inhibition does not contribute to plasticity with the stimulus strengths used in this study (Prescott 1997).

\section{DUAL-PROCESS THEORY OF LEARNING}

Interactions between learning processes are important in determining how plasticity develops and how it is expressed. At the synaptic level, the effects of depression influence whether spike broadening or vesicle mobilization is the predominant cause of facilitation (Klein 1995; Byrne and Kandel 1996). At the network level, Hawkins et al. (1998) have suggested recently that interactions between habituation and inhibition may underlie some of the differences between dishabituation and sensitization previously described in Aplysia (e.g., Marcus et al. 1988). Dual-process learning, characterized by transient sensitization followed by habituation (Groves and Thompson 1970), is also thought to result from interactions between learning processes at the network level (Prescott 1998). The plasticity exhibited by the tentacle withdrawal reflex is consistent with the dual-process theory of plasticity.

The results of this study support our hypothesis that the relative positioning of depression and facilitation affects how these forms of plasticity interact to determine the kinetics of dual-process learning. As stimulation is repeated, depression occurs at sensory neuron output synapses. With weak stimulation, depression is robust and occurs in the absence of facilitation. With stronger stimulation, depression still occurs but is weaker and slower to develop, consistent with the standard features of habituation (Thompson and Spencer 1966), and, moreover, the changes in synaptic transmission are confounded by the introduction of facilitation. Like depression, facilitation is expressed at an upstream locus, but it may not be ubiquitous in all neural pathways given its branch specificity (see above).

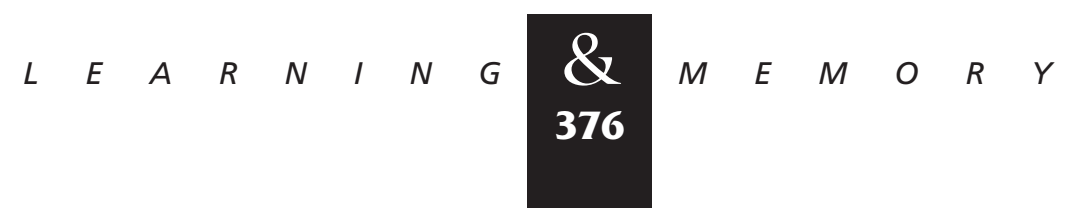


Unlike depression, which is by a homosynaptic process, facilitation is heterosynaptic and relies on facilitatory neurons for its induction. These facilitatory neurons are excited via sensory neurons whose output is prone to depression; therefore, the induction of sensitization will wane as depression develops upstream and causes reduced activation of facilitatory neurons. We refer to this reduction in sensitization's induction as the habituation of sensitization. Facilitation is also expressed upstream, but its pathway specificity is such that facilitation does not enhance the induction of facilitation, that is, there is no positive feedback loop (see Prescott 1998). Therefore, the relative positioning of plasticity in the network may explain the longstanding observation that sensitization tends to habituate (Lehner 1941; Thompson and Spencer 1966; Pinsker et al. 1970).

Our hypothesis is, therefore, that depression is expressed upstream of the site at which facilitation is induced and that the consequent interaction between the learning processes leads to the kinetics of dual-process learning. Hill and Jin (1998) have described a very similar pattern of plasticity in the cricket cercal system. When depression and facilitation occur together in that system, learning kinetics characteristic of dual-process learning are observed. Furthermore, all of the sensory neuron synapses display depression thought to be mediated by a presynaptic mechanism. Facilitation also occurs but only at a subset of synapses. The hypothesis is that facilitation is mediated by a retrograde signal from the postsynaptic cell (Davis and Murphy 1993). The important observation is that the same relative positioning of depression and facilitation as observed in the present paper gives rise to dual-process learning kinetics in the cricket cercal system.

Having not yet identified the facilitatory neurons in the tentacle withdrawal reflex, we have not been able to establish with certainty whether reduced input to facilitatory neurons is responsible for the habituation of sensitization in Helix. Reduced input to the facilitatory neurons would occur if innervation of those neurons were predominantly through the monosynaptic pathway; successful induction of sensitization by short-duration, high-intensity stimulation of the olfactory nerve (Fig. 8) is consistent with this type of innervation. Reduced input to central facilitatory neurons is also consistent with the observation that facilitation has a greater effect on peripheral pathways than on central ones (see Results). Hypothetically, depression of facilitatory neuron output could also cause the habituation of sensitization, but the observation that sensitization does not wane when the olfactory nerve is repeatedly stimulated (Fig. 8) argues against such a mechanism and further suggests that decremental changes are occurring upstream of the putative facilitatory neurons. The capacity to cause dishabituation by stimulation elsewhere than the tentacle (Prescott and Chase 1996), indicates that the capacity for incremental change is intact and, therefore, that depression predominantly affects the induction rather than the expression of facilitation. Dishabituation is, of course, an important criterion for habituation (Thompson and Spencer 1966), and it suggests that depression of facilitatory neuron output should not occur, but one must be cautious in equating the mechanisms of dishabituation and sensitization (as earlier papers did, e.g. Groves and Thompson 1970) given more recent findings (Marcus et al. 1988).

In a recent review of the dual-process theory of plasticity (Prescott 1998), a set of differential equations was presented with the intention of describing the kinetics of dual-process learning based on a logical interpretation of the interactions between learning processes. As shown in that paper, data previously available to test the model were less than ideal. Some of the experiments presented here were specifically designed to elicit habituation and sensitization separately and thereby allow determination of the kinetics of the individual learning processes by fitting the appropriate curves. The effects of pure habituation and pure sensitization can be described by simple differential equations:

Pure habituation:

$$
d E_{H} / d t=-\eta\left(E_{H}-E_{\text {min }}\right) .
$$

Pure sensitization:

$$
d E_{S} / d t=\sigma\left(E_{\max }-E_{S}\right)
$$

$E$ represents synaptic efficacy, and subscripts identify the learning process causing $E$ to change. For habituation, $E_{H}$ decreases at rate $\eta$ to minimum asymptote $E_{\text {min }}$. For sensitization, $E_{S}$ increases at rate $\sigma$ to maximum asymptote $E_{\max }$ (see Fig. 9, legend). Equations 1 and 2 can be combined to describe the habituation of sensitization:

Habituating sensitization:

$$
\begin{aligned}
d E_{H S} / d t= & \sigma \cdot E_{H}(t) / 100\left[\left(E_{\max }-100\right) E_{H}(t) / 100\right. \\
& \left.+100-E_{H S}\right] .
\end{aligned}
$$

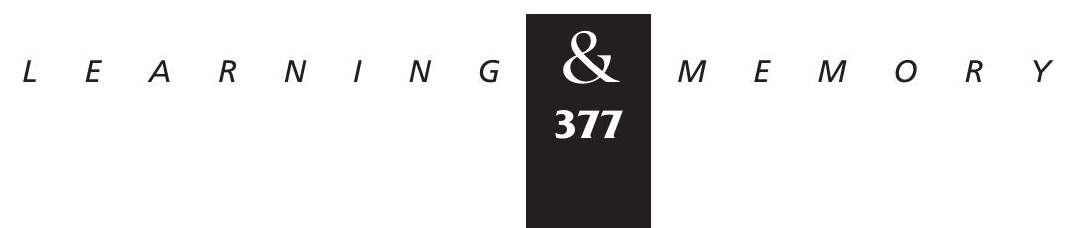




\section{Prescott and Chase}

Equation 3 is written such that the rate and extent of sensitization decrease as habituation develops. Equation 3 is slightly modified from its original form (Prescott 1998), but only to account for the different scale in which $E$ is expressed. The effects of habituating sensitization and habituation are added to determine the net plasticity given that depression and facilitation are most likely expressed in parallel in this system.

The numerical values for parameters in equations 1 and 2 were determined by fitting the curves for pure habituation and pure sensitization (Fig. 9). These values were then used in equation 3 to calculate the predicted plasticity associated with dualprocess learning. Comparison of the predicted plasticity with data from experiments in which de-

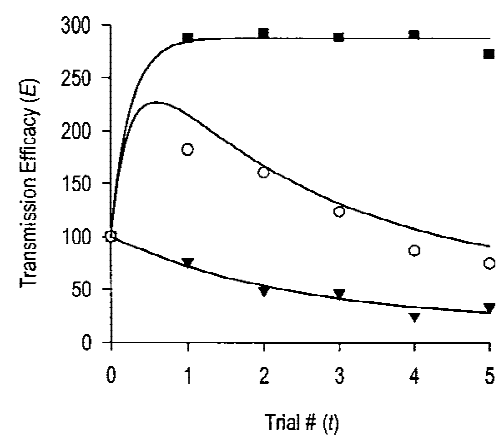

Figure 9: Comparison of empirical data with mathematical model for dual-process learning. Pure sensitization $\left(\boldsymbol{\square}, E_{s}\right)$ is represented here by plasticity in the late phase muscle response shown in Fig. 2C2; the data are fit $\left(r^{2}=0.99\right)$ by an exponential curve described by the differential equation $d E_{S} / d t=4.0\left(287.5-E_{S}\right)$. Pure habituation $\left(\boldsymbol{\Delta}, E_{H}\right)$ is represented by plasticity in the late phase muscle response shown in Fig. 4B2; the data are fit $\left(r^{2}=0.96\right)$ by the equation $d E_{H} / d t=-0.43$ $\left(E_{H}-19.5\right)$. The net plasticity resulting from concurrent habituation and habituating sensitization $\left(\bigcirc, E_{H S}+E_{H}\right.$ -100), that is, dual-process learning, is represented by plasticity in the late phase muscle response shown in Fig. 3B2; in this case, the curve is not a least squares fit of the data but, instead, represents the predicted plasticity using the numerical values from the above two curve fits in the equation for habituating sensitization, $d E_{H S} / d t=4.0 \cdot E_{H}(t) / 100\left[(287.5-100) E_{H}(t) / 100+\right.$ $\left.100-E_{H S}\right]$. This equation was numerically integrated using the fourth-order Runge-Kutta method to calculate $E_{H S}$ as a function of time, and the final net plasticity was determined as $E_{H S}(t)+E_{H}(t)-100$. The resultant curve describes the empirical data quite well $\left(r^{2}=0.84\right.$, which is significant, $P<0.05)$ suggesting that this model may accurately describe the nonlinear interactions between depression and facilitation that produce the kinetics characteristic of dual-process learning. pression and facilitation developed concurrently (Fig. 3B) shows that the simple mathematical model is reasonably successful in describing the kinetics of dual-process learning $\left(r^{2}=0.84\right.$; Fig. 9); this value of $r$ is significant $(P<0.05)$.

The apparent success of the model in dealing with the current data is not sufficient to validate the model, but it does demonstrate the model's utility for future research in this and other systems. For instance, if the neurons responsible for sensitization of the tentacle withdrawal reflex were identified, it would be desirable to determine the kinetics of depression in their input (or possibly output) for use in equation 3 , that is, to calculate the kinetics of the habituation of sensitization. If the prediction is correct that habituation of sensitization is faster and more robust than response habituation (Prescott 1998), the systematic overshoot of the predicted dual-process plasticity in Figure 9 would be eliminated. It would also be beneficial to test the model over a range of stimulus frequencies and intensities. This would evaluate how well the model predicts the kinetics of dual-process learning with quantitatively different combinations of depression and facilitation.

Findings from the current study illustrate the importance of identifying the location of plastic changes within a neural network. First, differential expression of plasticity in the various elements of the network can give rise to dissociative changes in the various components of behavior, such as the rate, peak amplitude, and duration of the response. Second, plasticity at a particular locus in a network can affect plasticity occurring elsewhere in the network giving rise to such phenomena as dual-process learning. Clearly, cellular plasticity must be considered at the network level if one wishes to fully explain the modification of behavior by learning.

\section{Acknowledgments}

This research was financially supported by a postgraduate scholarship to S.A.P. and an operating grant to R.C., both from the Natural Sciences and Engineering Research Council of Canada. We thank V. Castellucci, P. Drapeau, G. Pollack, and S. Ratté for their helpful advice. The publication costs of this article were defrayed in part by payment of page charges. This article must therefore be hereby marked "advertisement" in accordance with 18 USC section 1734 solely to indicate this fact.

\section{References}

Balaban, P. 1993. Behavioral neurobiology of learning in terrestrial snails. Prog. Neurobiol. 41: 1-19.

$$
\begin{array}{lllllllllllllll}
L & E & A & R & N & I & N & G & \underset{378}{\mathbf{Z}} & M & E & M & O & R & Y
\end{array}
$$


Brown, G.D. 1998. Nonassociative learning processes affecting swim probability in the seaslug Tritonia diomedea: Habituation, sensitization and inhibition. Behav. Brain Res. 95: 151-165.

Brown, G.D., W.N. Frost, and P.A. Getting. 1996. Habituation and iterative enhancement of multiple components of the Tritonia swim response. Behav. Neurosci. 110: $486-491$.

Byrne, J.H. and E.R. Kandel. 1996. Presynaptic facilitation revisited: State and time dependence. J. Neurosci. 16: 425-435.

Castellucci, V. and E.R. Kandel. 1976. Presynaptic facilitation as a mechanism for behavioral sensitization in Aplysia. Science 194: 1176-1178.

Castellucci, V., H. Pinsker, I. Kupfermann, and E.R. Kandel. 1970. Neuronal mechanisms of habituation and dishabituation of the gill-withdrawal reflex in Aplysia. Science 167: 1745-1748.

Christoffersen, G.R.J. 1997. Habituation: Events in the history of its characterization and linkage to synaptic depression. A new proposed kinetic criterion for its identification. Prog. Neurobiol. 53: 45-66.

Christoffersen, G.R.J., K. Frederiksen, J. Johansen, B.I. Kristensen, and L. Simensen. 1981. Behavioral modification of the optic tentacle of Helix pomatia; effect of puromycin, activity of S-100. Comp. Biochem. Physiol. 68: 611-624.

Clark, G.A. and E.R. Kandel. 1984. Branch-specific heterosynaptic facilitation in Aplysia siphon sensory cells. Proc. Natl. Acad. Sci. 81: 2577-2581.

Cleary, L.J. and J.H. Byrne. 1993. Identification and characterization of a multifunction neuron contributing to defensive arousal in Aplysia. J. Neurophysiol. 70: $1767-1776$.

Cohen, T.E., S.W. Kaplan, E.R. Kandel, and R.D. Hawkins. 1997. A simplified preparation for relating cellular events to behavior: Mechanisms contributing to habituation, dishabituation, and sensitization of the Aplysia gill-withdrawal reflex. J. Neurosci. 17: 2886-2899.

Davis, G.W. and R.K. Murphy. 1993. A role for postsynaptic neurons in determining presynaptic release properties in the cricket CNS: Evidence for retrograde control of facilitation. J. Neurosci. 13: 3827-3838.

Fischer, T.M. and T.J. Carew. 1993. Activity-dependent potentiation of recurrent inhibition: A mechanism for dynamic gain control in the siphon withdrawal reflex of Aplysia. J. Neurosci. 13: 1302-1314.

Fisher, S.A., T.M. Fischer, and T.J. Carew. 1997. Multiple overlapping processes underlying short-term synaptic enhancement. Trends Neurosci. 20: 170-177.

Frost, W.N. and E.R. Kandel. 1995. Structure of the network mediating siphon-elicited siphon withdrawal in Aplysia. J. Neurophysiol. 73: 2413-2427.

Frost, W.N., G.A. Clark, and E.R. Kandel. 1988. Parallel processing of short-term memory for sensitization in Aplysia. J. Neurobiol. 19: 297-334.

Groves, P.M. and R.F. Thompson. 1970. Habituation: A dual-process theory. Psychol. Rev. 77: 419-450.

Hawkins, R.D., V.F. Castellucci, and E.R. Kandel. 1981. Interneurons involved in mediation and modulation of gill-withdrawal reflex in Aplysia. I. Identification and characterization. J. Neurophysiol. 45: 304-314.

Hawkins, R.D., T.E. Cohen, W. Greene, and E.R. Kandel. 1998. Relationships between dishabituation, sensitization, and inhibition of the gill- and siphon-withdrawal reflex in Aplysia californica-effects of response measure, test time, and training stimulus. Behav. Neurosci. 112: 24-38.

Hill, A.A.V. and P. Jin. 1998. Regulation of synaptic depression rates in the cricket cercal sensory system. J. Neurophysiol. 79: 1277-1285.

Klein, M. 1995. Modulation of ion currents and regulation of transmitter release in short-term synaptic plasticity: The rise and fall of the action potential. Invert. Neurosci. 1: 15-24.

Lehner, G.F.J. 1941. A study of the extinction of unconditioned reflexes. J. Exp. Pyschol. 29: 435-456.

Lieb, J.R., Jr. and W.N. Frost. 1997. Realistic simulation of the Aplysia siphon-withdrawal reflex circuit: Roles of circuit elements in producing motor output. J. Neurophysiol. 77: 1249-1268.

Marcus, E.A., T.G. Nolen, C.H. Rankin, and T.J. Carew. 1988. Behavioral dissociation of dishabituation, sensitization, and inhibition in Aplysia. Science 241: 210-213.

Martin, K.C., A. Casadio, H. Zhu, E. Yaping, J.C. Rose, M. Chen, C.H. Bailey, and E.R. Kandel. 1997. Synapse-specific, long-term facilitation of Aplysia sensory to motor synapses: A function for local protein synthesis in memory storage. Cell 91: 927-938.

Menzel, R. and G. Bicker. 1987. Plasticity in neuronal circuits and assemblies of invertebrates. In The neural and molecular bases of learning (ed. J.-P. Changeux and M. Konishi), pp. 433-472. John Wiley and Sons, Toronto, Canada.

Pinsker, H., I. Kupfermann, V. Castellucci, and E.R. Kandel. 1970. Habituation and dishabituation of the gill-withdrawal reflex in Aplysia. Science 167: 1740-1742.

Prescott, S.A. 1997. "Interactions of habituation and sensitization at the network level illustrated by the tentacle withdrawal reflex of a snail." MSc thesis, McGill University, Montreal, Canada.

1998. Interactions between depression and

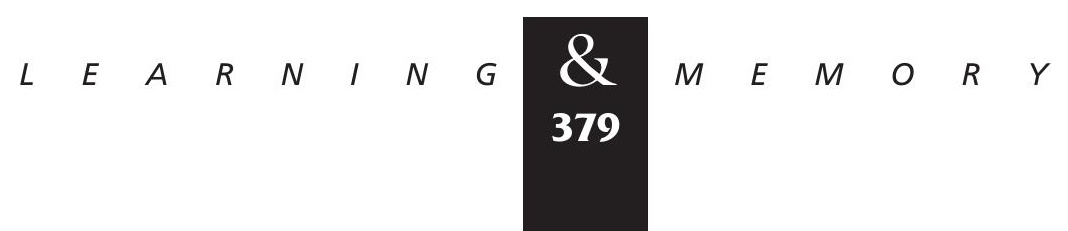




\section{Prescott and Chase}

facilitation within neural networks: Updating the dual-process theory of plasticity. Learn. \& Mem. 5: 446-466.

Prescott, S.A. and R. Chase. 1996. Two types of plasticity in the tentacle withdrawal reflex of Helix aspersa are dissociated by tissue location and response measure. J. Comp. Physiol. A 179: 407-414.

Prescott, S.A., N. Gill, and R. Chase. 1997. The neural circuit mediating tentacle withdrawal in Helix aspersa, with specific reference to the competence of the motoneuron C3. J. Neurophysiol. 78: 2951-2965.

Stopfer, M. and T.J. Carew. 1996. Heterosynaptic facilitation of tail sensory neuron synaptic transmission during habituation in tail-induced tail and siphon withdrawal reflexes of Aplysia. J. Neurosci. 16: 4933-4948.

Thompson, R.F. and W.A. Spencer. 1966. Habituation: A model phenomenon for the study of neuronal substrates of behavior. Psychol. Rev. 73: 16-43.

Trudeau, L.-E. and V.F. Castellucci. 1993a. Functional uncoupling of inhibitory interneurons plays an important role in short-term sensitization of Aplysia gill and siphon withdrawal reflex. J. Neurosci. 13: 2126-2135.

1993b. Sensitization of the gill and siphon withdrawal reflex of Aplysia: Multiple sites of change in the neuronal network. J. Neurophysiol. 70: 1210-1220.

Walters, E.T., J.H. Byrne, T.J. Carew, and E.R. Kandel. 1983. Mechanoafferent neurons innervating tail of Aplysia. I.

Response properties and synaptic connections. J.

Neurophysiol. 50: 1522-1542.

White, J.A., I. Ziv, L.J. Cleary, D.A. Baxter, and J.H. Byrne. 1993. The role of interneurons in controlling the tail-withdrawal reflex in Aplysia: A network model. J. Neurophysiol. 70: 1777-1786.

Zakharov, I.S. and P.M. Balaban. 1987. Neural mechanisms of age-dependent changes in avoidance behaviour of the snail Helix lucorum. Neurosci. 23: 721-729.

Received July 30, 1998; accepted in revised form April 16, 1999 


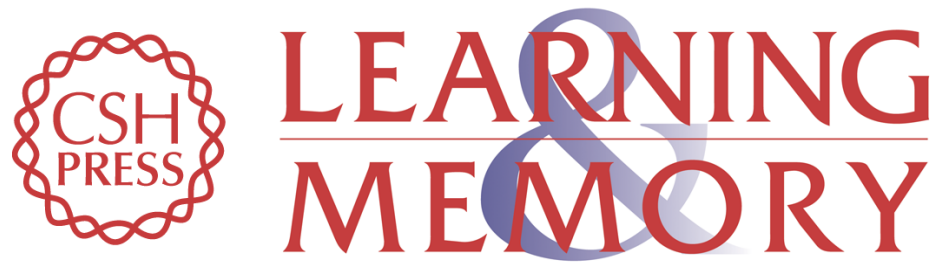

\section{Sites of Plasticity in the Neural Circuit Mediating Tentacle Withdrawal in the Snail Helix aspersa: Implications for Behavioral Change and Learning Kinetics}

Steven A. Prescott and Ronald Chase

Learn. Mem. 1999, 6:

Access the most recent version at doi:10.1101//m.6.4.363

References This article cites 35 articles, 12 of which can be accessed free at: http://learnmem.cshlp.org/content/6/4/363.full.html\#ref-list-1

License

Email Alerting

Receive free email alerts when new articles cite this article - sign up in the box at the Service top right corner of the article or click here. 